\title{
Application of the Photon Doppler Velocimetry (PDV) technique in tension-torsion Hopkinson bar experiments
}

\author{
Lukasz Farbaniec ${ }^{1}$, Yuan Xu $\mathrm{Xu}^{1}$, Junyi Zhou ${ }^{1}$, Sophoclis Patsias ${ }^{2}$, Duncan Macdougall ${ }^{2}$, \\ Julian Reed ${ }^{2}$, Nik Petrinic ${ }^{1}$, Clive Siviour ${ }^{1}$, Antonio Pellegrino ${ }^{1}$, and Daniel E. Eakins ${ }^{1}$ \\ ${ }^{1}$ Department of Engineering Science, University of Oxford, Parks Road, Oxford OX1 3PJ, UK \\ ${ }^{2}$ Rolls-Royce PLC, PO Box 31, Derby, DE24 8BJ, UK
}

\begin{abstract}
The Photon Doppler Velocimetry (PDV) technique is used to capture simultaneously propagating elastic waves of longitudinal and shear nature in a Tension-Torsion Hopkinson Bar (TTHB) apparatus. The system uses a pair of probes per velocity measurement, which were taken on the opposite sides of the TTHB bar with a laser irradiated spot size of $\sim 35 \mu \mathrm{m}$. The collected data were compared to the measurements obtained from the conventional strain gauge technique, and were in good agreement. The PDV method was effective in separating longitudinal and rotation signals even when they were superimposed on each other at the gauge location. This approach is also shown to be effective in detecting and accounting for the presence of bending waves in the TTHB bars.
\end{abstract}

\section{Introduction}

The split-Hopkinson pressure bar apparatus is a standard and widely used testing method for characterizing the high strain-rate behaviour of materials [1-3]. In the most common arrangement, the specimen is loaded by an incident pulse generated by an impact or a sudden release of stored energy traveling along the bar, where it is measured by a set of strain gauges. By assuming that the bars remain elastic during the test, the forces and velocities applied to the specimen can be found from the strain signals using an appropriate wave theory. The reliability and accuracy of the strain gauge depends on its size, type and the frequency response of the instrumentation. That is, it is impossible to resolve the strain wave features smaller than the active gauge length (characteristic frequency response) or frequencies outside the range of the driver/amplifier [4]. Another commonly reported limitation of the strain gauge technique is that the data is sensitive to electromagnetic interference produced by other circuits and devices [5]. In practice, reliable strain measurements also require well calibrated strain gauge sensors and good adhesion of the gauges to the surface of the bars. This is because an impact event can affect the strength of bonding between the strain gauges and the bars, and/or even break electrical connections (thus, abruptly terminating the measurements). Finally, the application of strain gauges becomes even more challenging with the progressive miniaturization of dynamic testing methods [6]. It is therefore of great importance to investigate other and more robust techniques for the measurement of strain signals and/or direct measurements of bar velocities. 
The photonic Doppler velocimetry (PDV) technique is commonly used in the domain of high energy physics focused on explosive and shock events [7-8]. It is based on a 1-D Fourier transform analysis of a heterodyne laser interferometry signal and can provide accurate measurements of the velocity of materials subjected to shock, impact and blast scenarios. Although SHPB experiments explore a material's behaviour at much lower rates of loading, the PDV technique has been successfully applied to track the rapidly varying low velocities of the SHPB bars or to measure the surface changes of specimens during such tests. Lo et al. (1997) performed pioneering experiments demonstrating particle velocity measurements on the free surface end of the SHPB bar using the all-fiber Doppler velocimeter [9]. The measurements of velocities at the sides of the bars, the radial expansion of the specimen, as well as the striker bar velocity, were subsequently explored in other studies [10-13]. More recently, Casem et al. (2013) showed that the PDV measurement of particle velocity can contain a significant component due to bending waves and proposed a method with multiple PDV measurements to account for this effect [14]. Other related studies demonstrated the application of optical measurement techniques to miniaturised SHPB systems with bars having a diffraction grating [15], or the use of temporal speckle interferometry in-plane displacement measurement devices to replace the strain gauges in the SHPB tests [16].

This study expands on previous works by demonstrating the use of the PDV technique as an effective way of capturing simultaneously propagating elastic waves of longitudinal and shear nature along the bars in a Tension-Torsion Hopkinson Bar (TTHB) apparatus.

\section{Experimental Methods}

A schematic of the TTHB system used in this study is shown in Fig 1a. The system consisted of two $\mathrm{Al}$ bars having a diameter and length of $20 \mathrm{~mm}$ and $2.5 \mathrm{~m}$, respectively. The tension and shear strain energies were prestored and released by a single clamp mechanism positioned in proximity to the end of the input bar. The torque and tensile forces were applied upon the other end of the input bar through a system of actuators. The clamp was released by breaking a notched pin with increasing clamping force. The bars were connected together using an adapter of the same material and diameter, so that a part of the stored energies travelled directly into the output bar. The propagation of corresponding tensile and shear waves was captured by the strain gauges and the PDV system at different locations on the bars, as shown in Fig. 1a-b. More details about the development of the TTHB system can be found in $\mathrm{Xu}$ et al. (2020) [17].

(a)

(a) torque
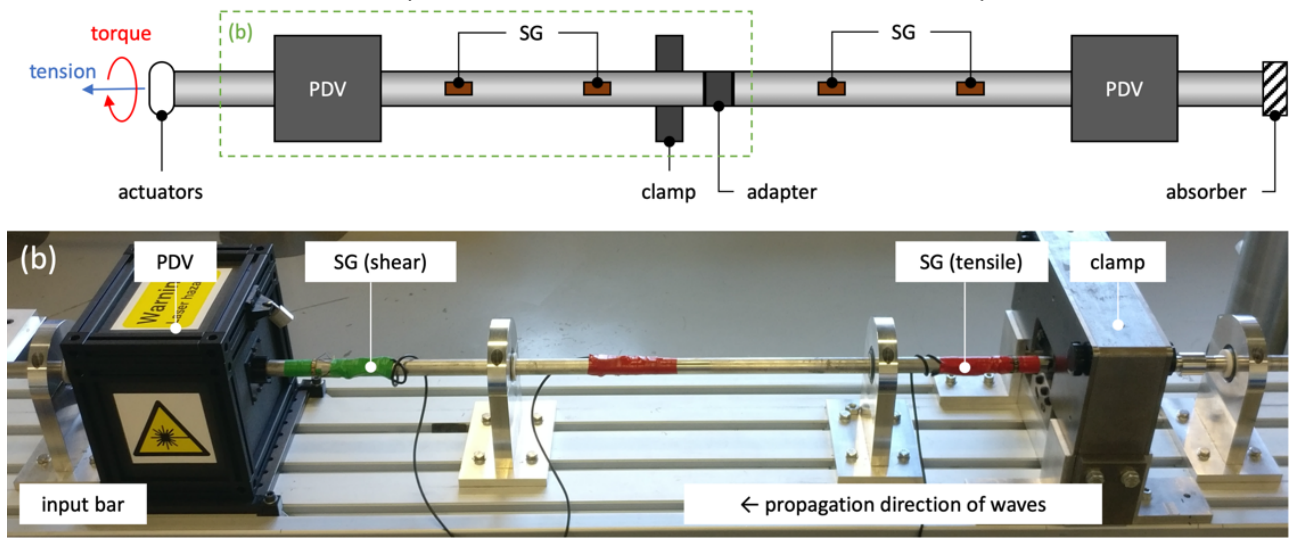

Fig. 1.: (a) Schematic of the TTHB system; (b) input bar of the TTHB system with the indicated locations of the strain gauges (SG) and the PDV enclosure. 
A schematic representation of a single PDV channel is shown in Fig. 2a. The PDV system, by using a heterodyne technique, obtains the heterodyne beat signal by mixing a reference signal $\left(f_{0}\right)$ and a Doppler shifted reflection of $f_{b}(t)$ off a moving bar surface. The resulting beat frequency, $\Delta f_{b}(t)=f_{b}(t)-f_{0}$, is related to the surface velocity, $u(t)$, by

$$
u(t)=\frac{\lambda_{0}}{2} \Delta f_{b}(t)
$$

where $\lambda_{0}$ is the wavelength of probing laser.

The source of two signals are narrow line-width $(<700 \mathrm{~Hz})$ single-frequency fibre laser sources operating at $1550 \mathrm{~nm} \mathrm{CW}$. The detector used for the beat frequency measurements was a fibre input InGaAs biased detector (Thorlabs DET08CFC/M), and the signal from this detector was captured by a LeCroy WaveMaster $808 \mathrm{Zi}-\mathrm{B}$ oscilloscope having a bandwidth of $8 \mathrm{GHz}$ and a sampling rate of $40 \mathrm{GS} / \mathrm{s}$. The system was capable of recording up to 8 optical channels simultaneously ( 2 oscilloscopes). The oscilloscopes were set to trigger and acquire data when the tensile stress wave (incident wave) has reached the strain gauge. The fibre probes were custom-made pigtail style collimators for $1550 \mathrm{~nm}$ with a focal length of $30 \mathrm{~mm}$ and a spot size of $35 \mu \mathrm{m}$. Three pairs of probes were installed on the input and output bar such that the measurements of longitudinal motion (indicated as L1 \& L2 in Fig. 2b), rotational motion (indicated as R1 \& R2 in Fig. 2b) and transverse motion (indicated as T1 $\& \mathrm{~T} 2$ in Fig. 2b) of the bars could be captured. The measurements were made on opposite sides of the bars to minimize disturbances in the measured signals originating from the propagation of bending waves. For transverse motion measurements, the probes were arranged normal to the surface of the bar. In other cases, the laser beams irradiated the bar at an incidence angle of $\theta=35^{\circ}$ with respect to the surface, as shown in Fig. $2 \mathrm{~b}$.
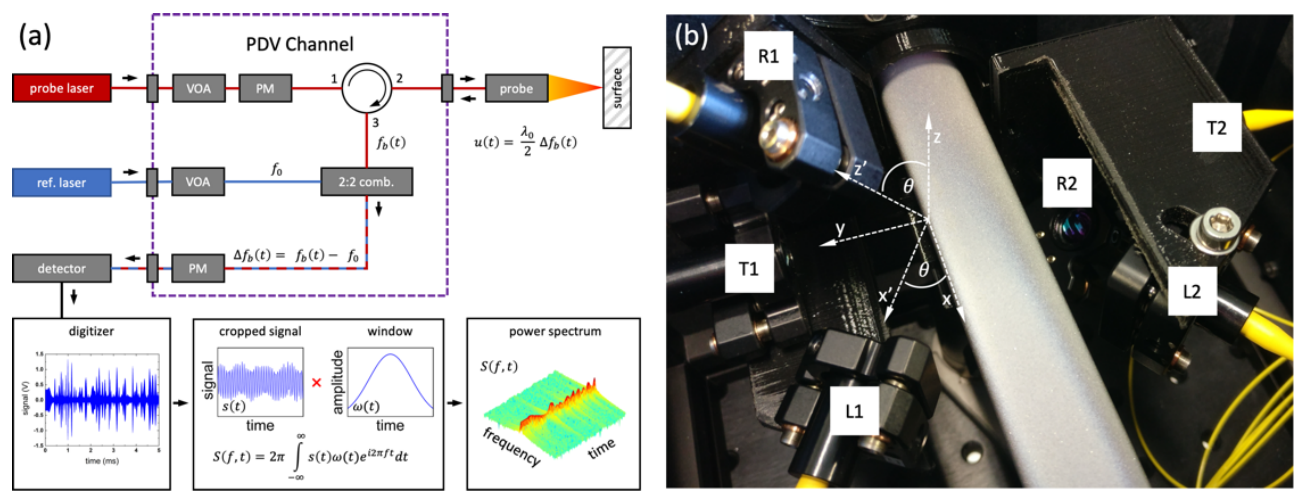

Fig. 2.: (a) Schematic of the PDV channel and the data analysis process; (b) three pairs of probes located inside each PDV enclosure for the measurements of longitudinal (L1 \& L2), rotational (R1 \& R2) and transverse (T1 \& T2) motions, respectively.

The captured interference patterns were consequently split into a series of overlapping time windows, and Fourier transformed to create a series of spectra (Fig. 2a). The surface velocities were retrieved by fitting a Gaussian curve to the centre value of the peak in each vertical segment of the power spectrum. Signals away from the peak velocities were filtered and avoided during fitting. Further details about the STFT data analysis process can be found in Strand et al. [7]. The spectrogram was obtained by using window lengths and overlap ratio of $10 \mu \mathrm{s}$ (50 000 data points) and 0.75 , respectively. The true velocity $\left(V_{\text {true }}\right)$ was calculated by averaging the velocity measurements made on the opposite side of the bar and accounting for the projection of the bar axis onto the laser axis as,

$$
v_{\text {true }}=\frac{<v_{\text {meas. }}>}{\cos \theta}
$$

where $\theta$ is the incidence angle of probing laser. 
For the strain gauges, the tensile/shear strain within the bar can be expressed as

$$
\varepsilon=\frac{i U_{0}}{G_{F} U_{I}}
$$

where $U_{0}$ is the voltage output of the Wheatstone bridge, $U_{I}$ is the excitation voltage, $G_{F}$ is the gauge factor (sensitive to the prestored tensile and shear energies if applied together), and $i=2,4$ and depends on the circuit configuration.

The tensile/shear strain within the bar and the velocity in the elastic region are related by

$$
\varepsilon=\frac{v}{c_{t, s}}
$$

where $v$ is the velocity, and $c_{t, s}$ is the tensile/shear speed of sound in the bar.

\section{Results and Discussion}

Figure $3 a-b$ shows the velocity measurements made by the three pairs of probes located on the input bar. First, it is worth noting the velocity measurements made by the probes T1 and $\mathrm{T} 2$. In the ideal case scenario, the input bar moves/rotates along the symmetry axis, and these two probes should measure no velocity change. The non-zero velocity measurements denote the presence of bending waves in the bar, which likely originated from the clamp release mechanism. Consequently, this undesirable motion of the bar caused disturbances in the measured signals by the other probes. Note that the longitudinal velocity measurements show a good agreement between the signals measured by the probes located on the opposite side of the bar (L1 and L2) until the arrival of bending waves (Fig. 3a). The reason for the disturbances in the measured signals occurring at the later stage is that the bending waves travel at a slower speed than the longitudinal waves. The input bar, by moving towards the probe T1, added a positive velocity component in the measurements made by the probe L1. The opposite effect was observed on the other side of the bar. However, such interference associated with bending waves is usually eliminated when the average of both signals is used in the further analysis, as is the case with the strain gauge measurements.
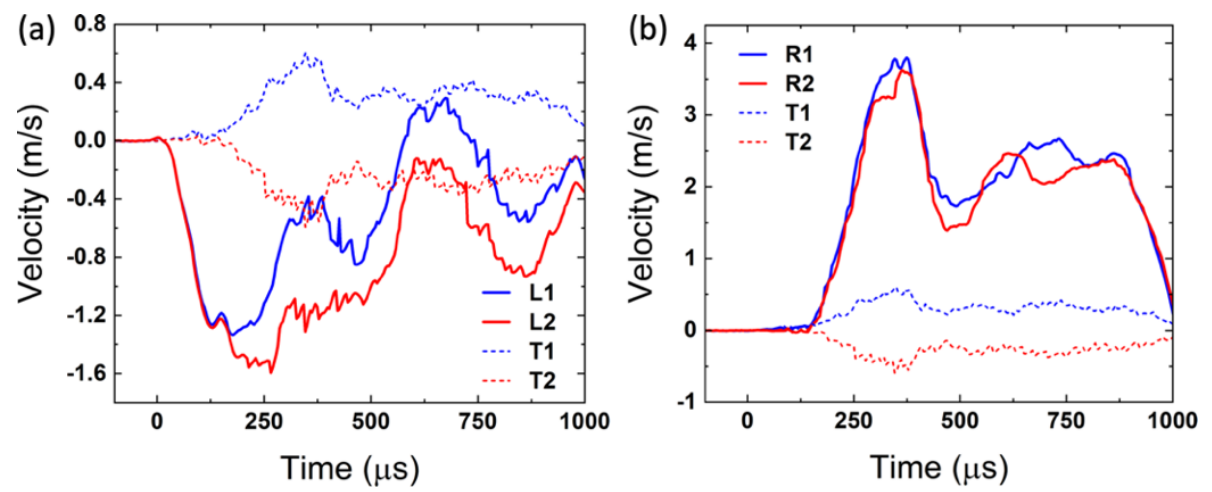

Fig. 3. PDV velocity measurements made by the three pairs of probes located on the input bar, where: (a) L1 and L2 are the longitudinal velocity profiles, and T1 and T2 are the transverse velocity profiles,

(b) R1 and R2 are the rotational velocity profiles, and $\mathrm{T} 1$ and $\mathrm{T} 2$ are the transverse velocity profiles.

The release of the prestored tension strain energy moved the input bar away from the probes that measured the longitudinal velocity and hence, the measured velocity was negative. In the case of rotational velocity measurements, the bar was rotating towards both probes, and the measured velocity has a positive sign (Fig. 3b). It should be noted that the bending waves are dispersive in nature (different frequencies travel at different speeds), thus the disturbances caused by their presence was observed along the whole shear wave 
pulse. The bending waves contributed to the velocity measurements made by the probes R1 and R2, and consequently must be considered in the further analysis.

Figure $4 \mathrm{a}-\mathrm{b}$ shows a comparison of measured velocities of the input bar by the two techniques. There is a good agreement between the collected data, despite the presence of bending waves and that the measurements were taken at different location on the bar (see Fig. 1b). Small fluctuations in the magnitude of measured signals might have two origins. First, the PDV technique is more sensitive to the presence of bending waves. That is, if the bending wave is in a different plane, the bar is moving away from its initial position and measurements are no longer taken in the middle (reference) plane of the bar (the measured component of velocity has changed), while the strain gauge data is always collected from equally spaced 4 strain gauges bonded around the bar diameter. On the other hand, the strain gauges are sensitive to strains in directions other than the principal strain axis. For example, if only the tensile (or compression) strain energy is stored in the system, the loading direction remains constant with regard to the tensile strain gauge, and the collected data can be directly analysed and related to the measurements of longitudinal strain. However, the application of secondary rotational loading to the input bar can result in geometrical effects, such as twisting instability in the form of deflection or bending, and consequently affects the readings of the tensile strain gauge. Other effects, such as the classic Poynting effect might also add to the complexity of the system, since the rotational load applied to the $\mathrm{Al}$ bar results in its elongation [18-19].
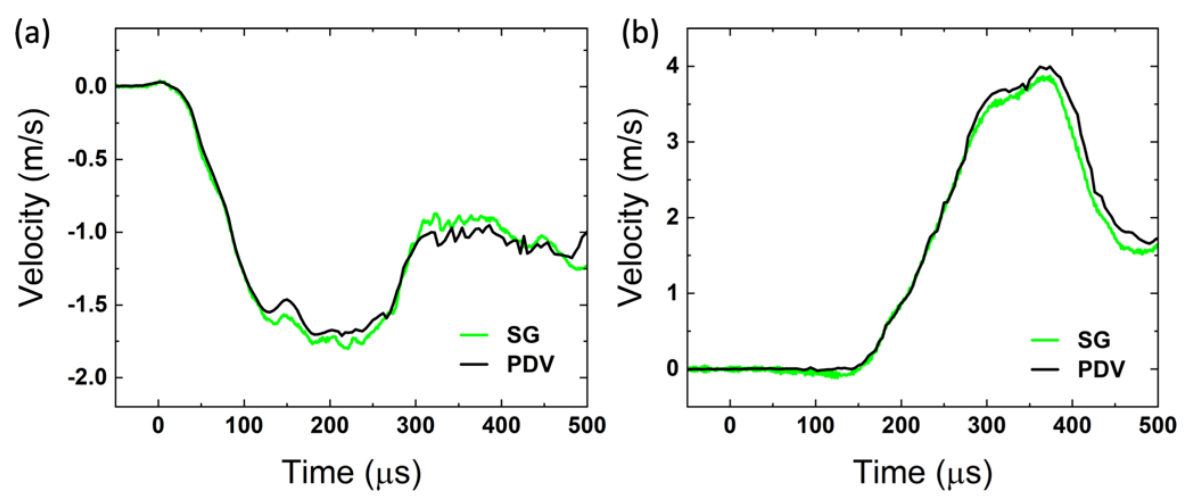

Fig. 4. Comparison of the longitudinal (a) and rotational (b) velocity profiles measured by the strain gauges (SG) and the PDV techniques.

Overall, the results show that the PDV technique is an attractive diagnostic tool for the TTHB experiments. Such an approach has been effective in separating longitudinal and rotation signals even when they were superimposed at the gauge location. One of the key advantages over the traditional strain gauge technique is that such laser-based approach is non-invasive and insensitive to the amplitude of incident pulses in the TTHB system. Thus, it allows for experiments at higher strain-rates with a reduced risk of data loss. The PDV systems has also a clear advantage over the strain gauge technique in the systems that use prestored energy to generate combined loading pulses. This is because the prestored energy in the form of combined loading (e.g., tension/compression and torsion) gives rise to geometrical effects like twisting instability or the Poynting effect, which require careful calibration to ensure the accurate strain measurements.

Another advantage of the PDV technique is that the data is recorded in the frequency domain of the measured signals. This makes the data insensitive to electrical noise, and more reliable than the strain gauge technique. The theoretical upper frequency (the $-3 \mathrm{~dB}$ point) for a bonded strain gauge was estimated to be approximately $600 \mathrm{kHz}$, while the PDV technique was limited by the instrumentation and electronic components used to evaluate it ( $5 \mathrm{GHz})$. 
Also, the PDV measurements were made at a discrete representation of the cross section (spot size of $35 \mu \mathrm{m}$ ) allowing for higher precision in measured strain-profiles, as compared to the strain gauges ( $2 \mathrm{~mm}$ gauge length). The PDV approach can therefore be applied to much smaller split-Hopkinson pressure bar apparatus, where the strain gauge measurements are challenging. Finally, the PDV technique as an active diagnostic tool can be used at any location on the TTHB system. For example, it can provide a direct measurement of the end bar velocity.

This work was conducted as part of the UK Engineering and Physical Sciences Research Council (EPSRC) Prosperity Partnership grant "Cornerstone: Mechanical Engineering Science to Enable Aero Propulsion Future" (EPSRC Reference: EP/R004951/1). The Impact Engineering Laboratory (University of Oxford) also acknowledges the support of Rolls-Royce PLC. Finally, the authors would like to thank Mr Stuart Carter and his team at the University of Oxford for their technical support.

\section{References}

1. H. Kolsky, Proc. Phys. Soc. B 62:676-700 (1949)

2. P.S. Follansbee, Metals Handbook, 8(9), American Society for Metals, Metals Park, OH, 198-217 (1985)

3. W.W. Chen, B. Song, Split Hopkinson (Kolsky) bar: design, testing and applications (Springer Science \& Business Media, 2010)

4. K. Ueda, A. Umeda., Exp. Mech., 38(2), 93-98 (1998)

5. A.D. Barr, S.D. Clarke, A. Tyas, J.A. Warren, Exp. Mech, 57(5), 813-817 (2017)

6. D. Casem, E. Huskins, J. Ligda, B. Schuster. A Kolsky bar for high-rate microcompression: Preliminary results. In Dynamic Behavior of Materials; Springer International Publishing: New York, NY, USA,; Volume 1, 87-92 (2016)

7. O.T. Strand, D.R. Goosman, C. Martinez, T.L. Whitworth, W.W. Kuhlow, Rev. Sci. Instrum., 77(8), 083108 (2006)

8. D.H. Dolan, Rev. Sci. Instrum. 91(5), 051501 (2020)

9. Y.L. Lo, J.S. Sirkis, W.L. Fourney, Exp. Mech., 37(3), 328-332 (1997)

10. C. Avinadav, Y. Ashuach, R. Kreif, Rev. Sci. Instrum., 82(7), 073908 (2011)

11. H. Song, X. Wu, C. Huang, Y. Wei, X. Wang, Rev. Sci. Instrum., 83(7), 073301 (2012)

12. X. Wu, X. Wang, Y. Wei, H. Song, C. Huang, Int. J. of Impact Eng., 69, 149-156, (2014)

13. L.J. Lea, A.P. Jardine, Rev. Sci. Instrum., 87(2), 023101, (2016)

14. D.T. Casem, M.B. Zellner, Exp. Mech., 53(8), 1467-1473 (2013)

15. D.T. Casem, S.E. Grunschel, B.E. Schuster, Exp. Mech., 52(2), 173-184 (2012)

16. S. Yang, Z. Gao, H. Ruan, C. Gao, X. Wang, X. Sun, X. Wen, Appl. Sci., 8(5), 808 (2018)

17. Y. Xu, L. Farbaniec, C. Siviour, D. Eakins, A. Pellegrino, The Development of Split Hopkinson Tension-Torsion Bar for the Understanding of Complex Stress States at High Rate, in Dynamic Behavior of Materials, Volume 1, Proceedings of the 2020 Annual Conference on Experimental and Applied Mechanics. Springer International Publishing (2021)

18. J.H. Poynting, Proc. Roy. Soc. London, Serie A, 82, 546-559 (1909)

19. B. Wack, Acta Mech., 80(1), 39-59 (1989) 